\title{
Diastolic Filling and Pressure Imaging: Taking Advantage of the Information in a Colour M-mode Doppler Image
}

\author{
S. De Mey ${ }^{1 *}$, J. De Sutter ${ }^{2}$, J. Vierendeels ${ }^{1}$ and P. Verdonck ${ }^{1}$ \\ ${ }^{1}$ Institute of Biomedical Technology, Ghent University, Ghent, Belgium; ${ }^{2}$ Department of Cardiology, \\ Ghent University Hospital, Ghent, Belgium
}

The ability to derive non-invasively information on left ventricular diastolic function on one hand and pressure gradients on the other hand, makes Doppler ultrasound a very attractive tool in clinical practice. However, the limitations of the standard Doppler approaches in differentiating between normal and pseudonormal filling patterns, together with the limitations of the simplified Bernoulli equation for assessing pressure gradients, are well described. In this manuscript the role of colour M-mode Doppler echocardiography as a tool that can overcome these limitations is discussed. Relevant key concepts of the haemodynamics of left ventricular filling and its relationship with colour M-mode Doppler echocardiography are introduced.

(Eur J Echocardiography 2001; 2: 219-233)

(C) 2001 The European Society of Cardiology

Key Words: colour M-mode Doppler, diastolic filling, flow propagation velocity, pressure imaging.

\section{Introduction}

\section{Background}

The potential ability to derive non-invasively information on left ventricular diastolic function, makes Doppler ultrasound a very attractive tool in clinical practice. The traditional approach to evaluate left ventricular diastolic function non-invasively uses pulsed wave Doppler echocardiography with the sample volume positioned at the level of the mitral valve leaflet tips. The recorded velocity profile of left ventricular

*Corresponding author: Stefaan De Mey, Hydraulics Laboratory, St-Pietersnieuwstraat 41, B-9000 Gent, Belgium. Tel: $\quad++32 / 9 / 2648927 ; \quad$ Fax: $\quad++32 / 9 / 2643595$. E-mail: stefaan.demey@navier.rug.ac.be

Received 28 May 2001; revised manuscript received 6 August 2001; accepted 8 August 2001. inflow shows an early filling wave (E-wave) and a late filling wave during atrial contraction (A-wave). A multitude of indices such as peak $E$ and $A$ velocity, their ratio, and acceleration and deceleration times are derived from this velocity pattern and proposed as markers for diastolic function ${ }^{[1-5]}$. Intensive research, using numerical ${ }^{[6-9]}$ and in vitro ${ }^{[10]}$ models, next to observations in animals and humans ${ }^{[8,11-16]}$, have clearly elucidated the physical and physiological determinants of the transmitral velocity pattern. Although it provided us with many clinical useful insights, this research revealed also the limitations of pulsed wave Doppler echocardiography in clinical practice for assessing diastolic function.

Another widely used application of Doppler echocardiography is the assessment of pressure drops across orifices: e.g. using the simplified Bernoulli equation pressure drops across the mitral valve are estimated based on pulsed wave Doppler derived velocities ${ }^{[17]}$. However, the limitations for assessing pressure gradients 


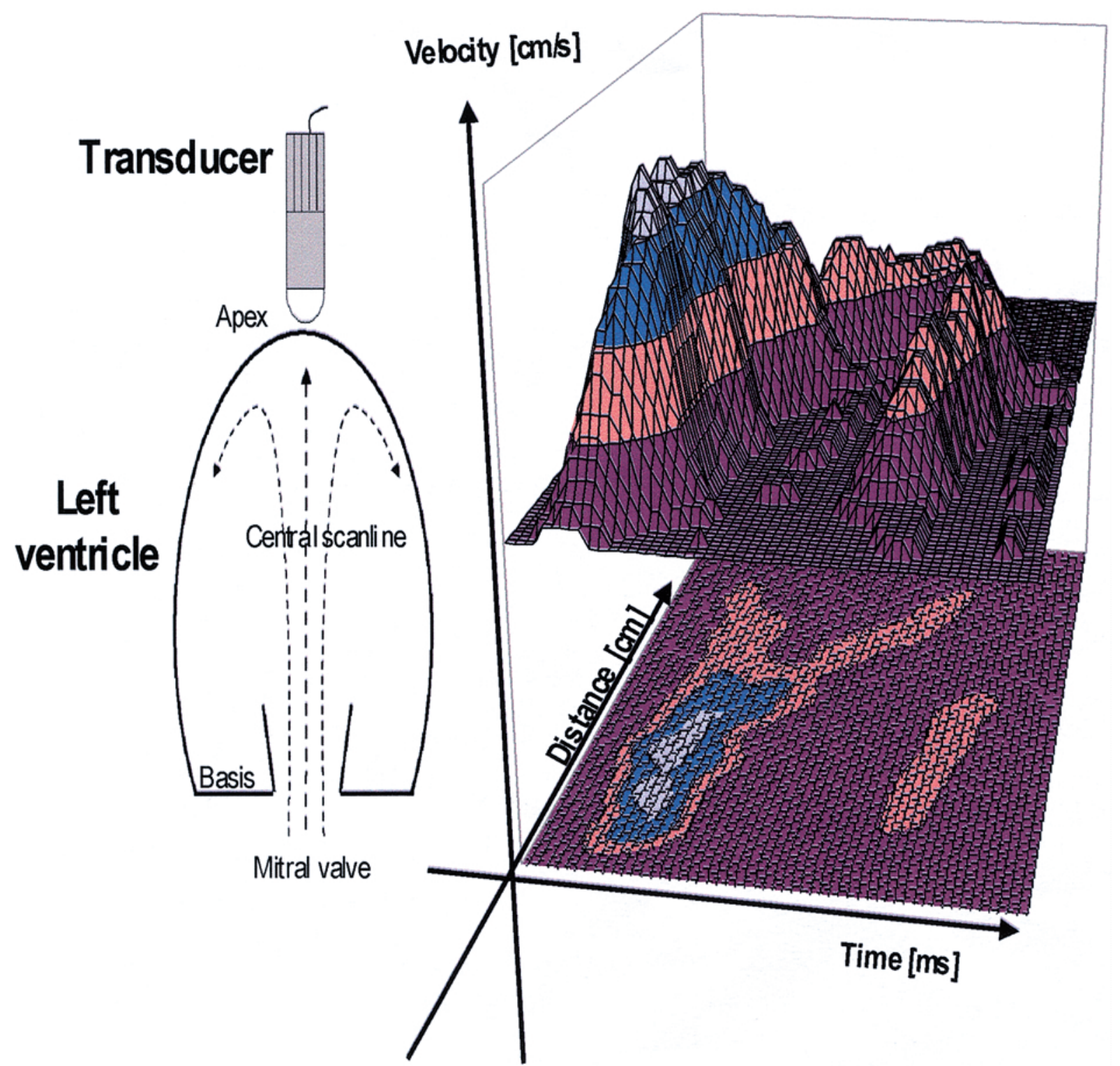

Figure 1. Left panel: Schematic representation of the recording of a colour M-mode Doppler image of left ventricular filling with the scanline aligned with the central inflow tract. Right panel: Spatio-temporal velocity information, obtained using colour M-mode Doppler echocardiography, represented in a three-dimensional graph (top) and in a two-dimensional plane with the velocity encoded in colors (bottom).

using the simplified Bernoulli equation are largely demonstrated $^{[18-21]}$.

To overcome the limitations of standard pulsed wave Doppler echocardiography, additional techniques remain necessary. Analysis of the pulmonary venous flow patterns ${ }^{[22]}$, and more recently the use of tissue Doppler imaging ${ }^{[23-27]}$, strain ${ }^{[28]}$ and strain rate imaging ${ }^{[29]}$, and colour M-mode Doppler echocardiography ${ }^{[30]}$ have been suggested as promising new tools in the evaluation of left ventricular diastolic function using ultrasound based technology. In this review we will focus on colour M-mode Doppler echocardiography.

\section{Colour M-mode Doppler Echocardiography of Left Ventricular Filling}

Whereas pulsed wave Doppler velocity measurements represent a temporal velocity variation throughout the cardiac cycle obtained at a single point in space, colour M-mode Doppler echocardiography interrogates all the velocities along a scan line and provides a spatiotemporal velocity map with a temporal resolution of $5 \mathrm{~ms}$, a spatial resolution of approximately $0.3 \mathrm{~mm}$ and a velocity resolution of typically $3 \mathrm{~cm} . \mathrm{s}^{-1[31]}$. Figure 1 (left panel) shows the orientation of the scan line, 
captured from an apical window. The scan line is parallel to the inflow track of left ventricular filling. The right panel (top) shows a three-dimensional graph containing the spatial and temporal distributed velocity information. A colour M-mode Doppler image provides the same three-dimensional information in a twodimensional plane with the velocity encoded in colours (Fig. 1, right panel, bottom).

Two distinct approaches are used to cope with the velocity information in a colour M-mode Doppler image. On the one hand, qualitative properties of the flow field within the left ventricular cavity are analysed. This approach has led to the concept of flow propagation velocity $\left(v_{p}\right)$. On the other hand, efforts are made towards the development of 'pressure imaging' techniques, i.e. visualizing and calculating pressure differences, thereby fully exploiting quantitatively the numerical velocity information, stored in a colour M-mode Doppler image. In this manuscript the progress in both approaches will be reviewed and discussed.

\section{Haemodynamics of Left Ventricular Filling and Colour M-mode Doppler Echocardiography}

\section{Haemodynamics of Left Ventricular Filling}

Colour M-mode Doppler echocardiography of left ventricular filling provides velocities along one scan line in a three-dimensional flow field. Therefore, to understand the haemodynamics of the velocity information in the colour M-mode Doppler image, the entire flow field within the left ventricular cavity has to be considered. From the early 1970 s to the late 1990 s, several research groups have focused their efforts on understanding the intra-ventricular flow field. Although those studies were conducted with different objectives, it provided us with many useful insights concerning the filling mechanisms of the left ventricle and confirmed that vortex formation is a crucial phenomenon in the mechanism of left ventricular filling.

Vorticity at a point in a fluid is related to the angular velocity of an infinitesimal fluid mass that is instantaneously occupying a point in space ${ }^{[32]}$. The injection of fluid through an orifice is a mechanism that generates vorticity, resulting in a smoke ring like vortex (Fig. 2, left panel). Therefore, because left ventricular filling is basically the filling of a cavity through an orifice, left ventricular filling generates smoke ring like vortices in the left ventricular cavity (Fig. 2, right panel). The diameter of the core of the ring (a) and the diameter of the annulus around the core (b) characterize the vortex ring as shown in Fig. 2. The fluid particles trapped within the annulus around the core are travelling with the vortex ring at a propagation speed $v_{p}$. This propagation speed does not equal the velocity of an individual fluid particle $(v)$ because individual fluid particles are rotating within the travelling vortex ring ${ }^{[32,33]}$.
The generation and travelling of vortices in the left ventricle is confirmed in several studies. In the 1970s, studies were initiated to investigate the influence of the flow field on valvular mechanics. Lee et al. ${ }^{[34]}$ with the use of a numerical model, and Bellhouse et al. ${ }^{[35]}$ with the use of an in-vitro model, described vortex formation during left ventricular filling. According to Bellhouse et $a l . .^{[35]}$, the presence of a vortex ring provides a mechanism for valve closure at the end of diastole. In the late 1980s and early 1990s several clinical studies have examined the spatial dependent differences of the intra-ventricular flow in the diseased left ventricle during the cardiac cycle ${ }^{[36-38]}$. Simultaneously, numerical and in vitro simulations provided a theoretical framework, explaining the observations ${ }^{[39,40]}$. From this research $^{[36-38]}$ it was concluded that travelling vortices especially are a feature of left ventricles that have become pathologically dilated. Also, a positive relationship between the presence of vortex rings and a risk of thrombus formation was suggested. However, the use of two-dimensional colour Doppler (which has a poor time resolution) and pulsed wave Doppler echocardiography (which provides information at only one location) for observing the subtle spatial differences during diastolic filling is a limitation in these studies. In contrast with those studies, recently Rodevand et al. ${ }^{[41]}$ described the presence of vortices in normal human left ventricles during diastolic filling using colour M-mode Doppler echocardiography, which has a superior time resolution compared with two-dimensional echocardiography.

In the 1990s, in vitro and numerical studies of the flow in a skeletal muscle left ventricle were very elusive in explaining the mechanism of vortex formation and travelling in a cavity ${ }^{[42-44]}$. In the in-vitro experiments, detailed information about the formation and travelling of vortices during filling was obtained with the use of a helium-neon laser that illuminated planar sections of the model ventricles. Influences of geometrical properties, including cavity volume, valve size, ejection fraction, and inclination of the mitral valve plane on vortex formation and travelling were investigated.

\section{Travelling Vortices and Colour M-mode Doppler Echocardiography of Left Ventricular Filling}

In the above mentioned experimental work $^{[42-44]}$, the positive relationship between the position of the vortex ring in the cavity and the position of the maximal blood velocity in the left ventricle along the base-apex axis, i.e. the fact that propagation speed of the vortex ring equals the propagation speed of the maximum velocity, was a key observation. Steen et al. ${ }^{[45]}$, using a hydraulic model, were the first to realize that the travelling speed of a vortex ring in the left ventricle could therefore be associated with the $v_{p}$ obtained from a colour M-mode Doppler image. This concept is illustrated in Fig. 3, showing two snapshots of the filling of a model left 

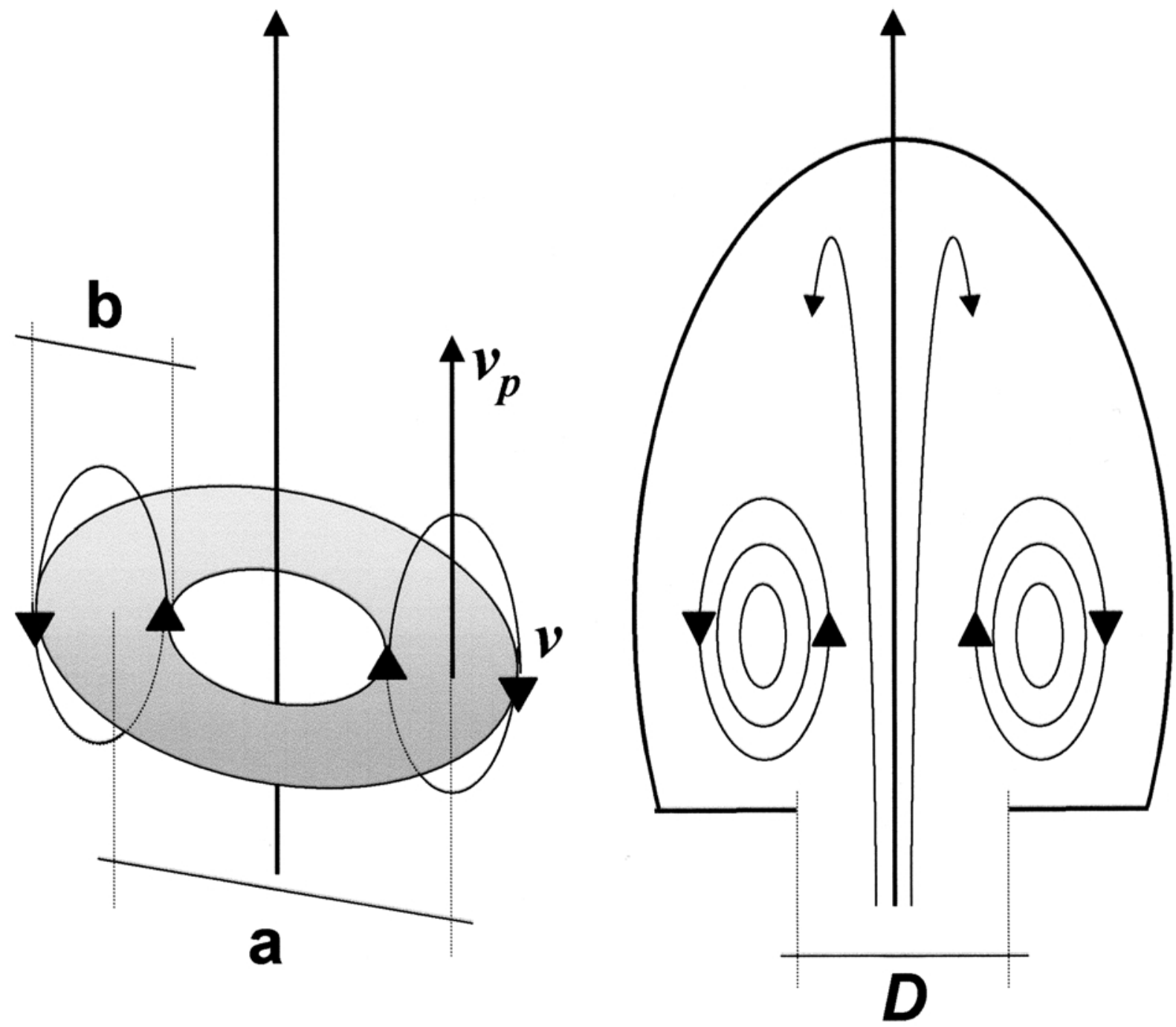

Figure 2. Principle of vortex formation during left ventricular filling. A smoke ring like vortex (left panel) with (a) the diameter of the core and (b) the diameter of the annulus around the core is formed in a left ventricle (mitral valve diameter $D$, right panel). The vortex is propagating with a propagation speed $v_{p}$ in the direction of the apex. Individual fluid particles are rotating within the vortex ring and have a velocity $v$.

ventricle next to a drawing of a corresponding colour M-mode Doppler image. Simultaneously with the propagation of the vortex ring from base (at time $t_{1}$ ) towards the apex (at time $t_{2}$ ), the maximum velocity is shifting upwards in the colour M-mode Doppler image. Therefore, the propagation speed of the ring vortex $v_{p}$ can be derived from the slope in the colour M-mode Doppler image:

$$
v_{p}=\frac{s_{2}-s_{1}}{t_{2}-t_{1}}
$$

Vierendeels et al. ${ }^{[46]}$, using a numerical model, confirmed this close relationship between the position of the vortex core and the position of the maximum velocities along the base-apex axis.

In case of vortex formation during left ventricular filling, peak $E$-velocity should always exceed $v_{p}$ because of the hydrodynamic principle that, due to the intrinsic circular motion of the particles in the ring vortex, the velocity of particles $(E)$ in a vortex ring exceeds the velocity at which the whole ring travels $\left(v_{p}\right)$. The higher peak $E$-velocity, compared to $v_{p}$, is observed in vitro and in numero ${ }^{[42-46]}$, and in vivo ${ }^{\left[47^{p}-49\right]}$.

\section{Biphasic Filling Pattern}

As described above, vortex formation is a crucial phenomenon in the filling process. However, during the initial flow phase, before the vortex ring has fully developed, blood moves almost simultaneously in the whole ventricle. Therefore, in a colour M-mode Doppler image of the early filling wave, very often a biphasicfilling pattern is observed. This is illustrated in Fig. 4, which shows a colour M-mode Doppler image of left 


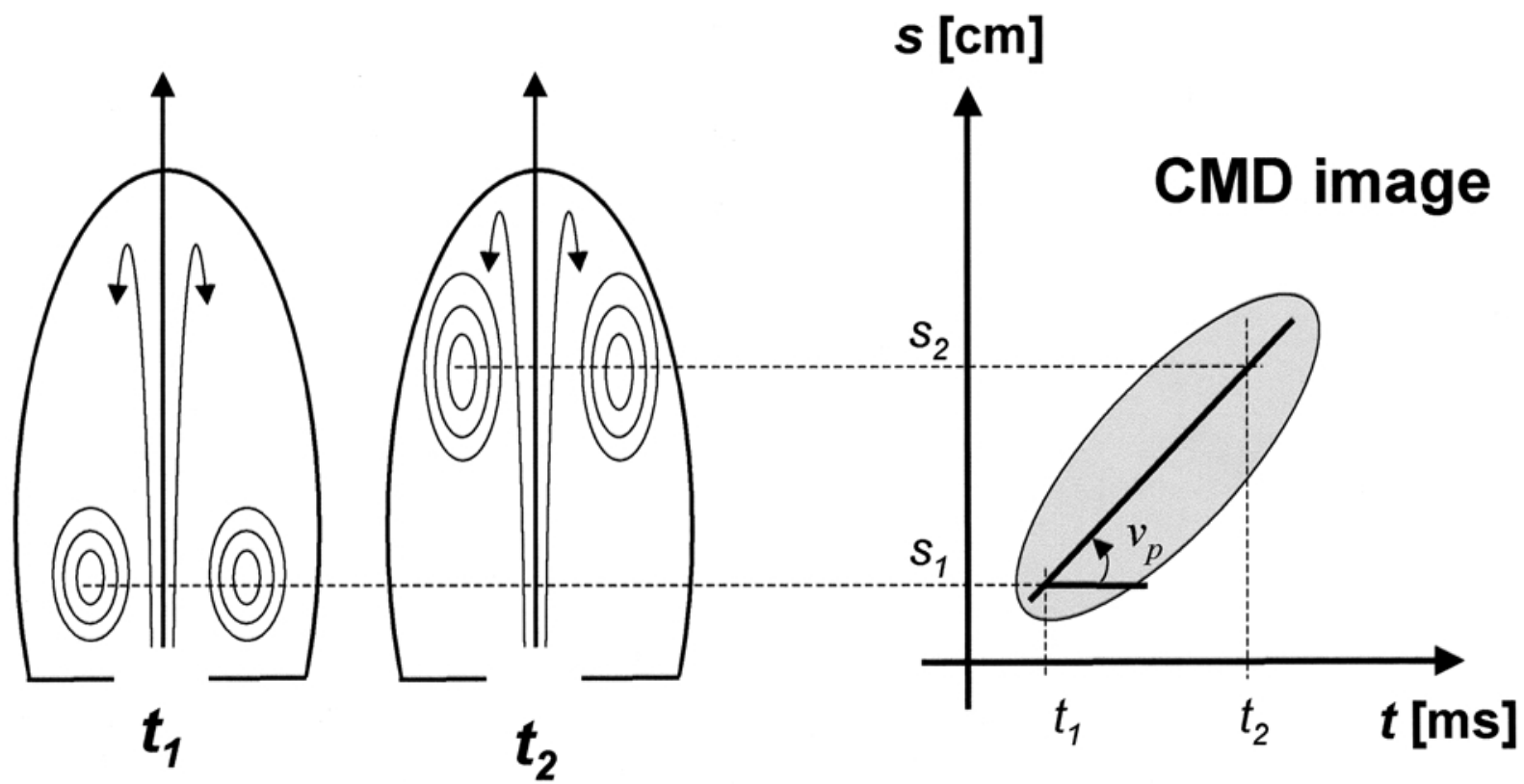

Figure 3. Physical interpretation of the flow propagation velocity as derived from a colour M-mode Doppler image. The position of the vortex ring at different moments in time in the left ventricular (left panel) correspond to the position of the maximal velocity along the base-apex axis, as obtained with colour M-mode Doppler echocardiography (right panel). Therefore the propagation speed of the vortex ring corresponds to the flow propagation velocity $\left(v_{p}\right)$, obtained from a colour Doppler M-mode image.

ventricular filling obtained in a normal subject. Phase I is straight upward and can be associated with the initial motion of the blood column in the left ventricle due to the passing pressure wave. Phase II is the propagation of the maximum velocity that can be associated with the propagation of a vortex ring as described before. The biphasic filling pattern is observed in vitro ${ }^{[45]}$, in numero ${ }^{[46]}$ and in vivo ${ }^{[30,41,49,50]}$.

\section{Assessment of Flow Propagation Velocity Using Colour M-mode Doppler Echocardiography}

\section{Quantifying $\mathrm{v}_{\mathrm{p}}$ from a Colour M-mode Doppler Image}

An adequate recording of a colour M-mode Doppler image of left ventricular filling starts with placing the M-mode line parallel with the direction of flow observed by two-dimensional colour Doppler echocardiography in an apical four-chamber view. Basically five different approaches are used for identifying $v_{p}$ of left ventricular filling using colour M-mode Doppler echocardiography manually or in a (semi) automated way. Differences between methods are mainly related to the amount of velocity information and/or the specific part of the colour M-mode Doppler image that is considered for identifying a line segment.

Method 1: Brun. Brun et al. ${ }^{[47]}$ defined $v_{p}$ as the slope (in $\mathrm{m} . \mathrm{s}^{-1}$ ) of the line segment identifying the black-to-colour transition of the early filling wave. The segment begins with the onset of flow in the left ventricular inlet and ends as far as possible in the left ventricular chamber. The line segment has to be reasonably linear [Fig. 5(a)].

Method 2: Duval-Moulin-Garcia. As the black-tocolour transition, as defined by Brun et al., is not always clearly visible, Duval-Moulin et $a l^{[51]}$ and Garcia et $a{ }^{[30]}$ have modified this method by considering boundaries of equal velocities, so-called isovelocity boundaries instead of black-to-colour transitions [Fig. 5(b)]. Typically the isovelocity contour of the first aliasing velocities (in case of manual analysis) or the isovelocity contour of $50 \%$ of the maximum velocity (in case of semiautomatic analysis) is addressed, starting at the position of the mitral valve, going typically $4 \mathrm{~cm}$ into the left ventricle.

Method 3: Stugaard. Stugaard et al. ${ }^{[49]}$ measured the occurrence of the maximal velocities at several positions on the left ventricular base-apex axis. The time delay $(T D$, in $\mathrm{ms})$ between occurrence of maximal velocity at the mitral leaflet tips and the maximal velocity further in the left ventricle (typically $37 \mathrm{~mm}$ away from the leaflet tips) is proposed as a characteristic for flow propagation [Fig. 5(c)]. The ratio $T D$ and the spatial distance $(S$, in $\mathrm{cm})$ between the first and last position is proposed as a normalized characteristic for $v_{p}$. The dimension of this normalized characteristic is the reciprocal of a velocity $\left(\mathrm{ms} . \mathrm{cm}^{-1}\right)$.

Method 4: Takatsuji. Takatsuji et al. ${ }^{[52]}$ determined the $v_{p}$ using the baseline shift, displaying the flow velocities higher than the aliasing velocities in blue 


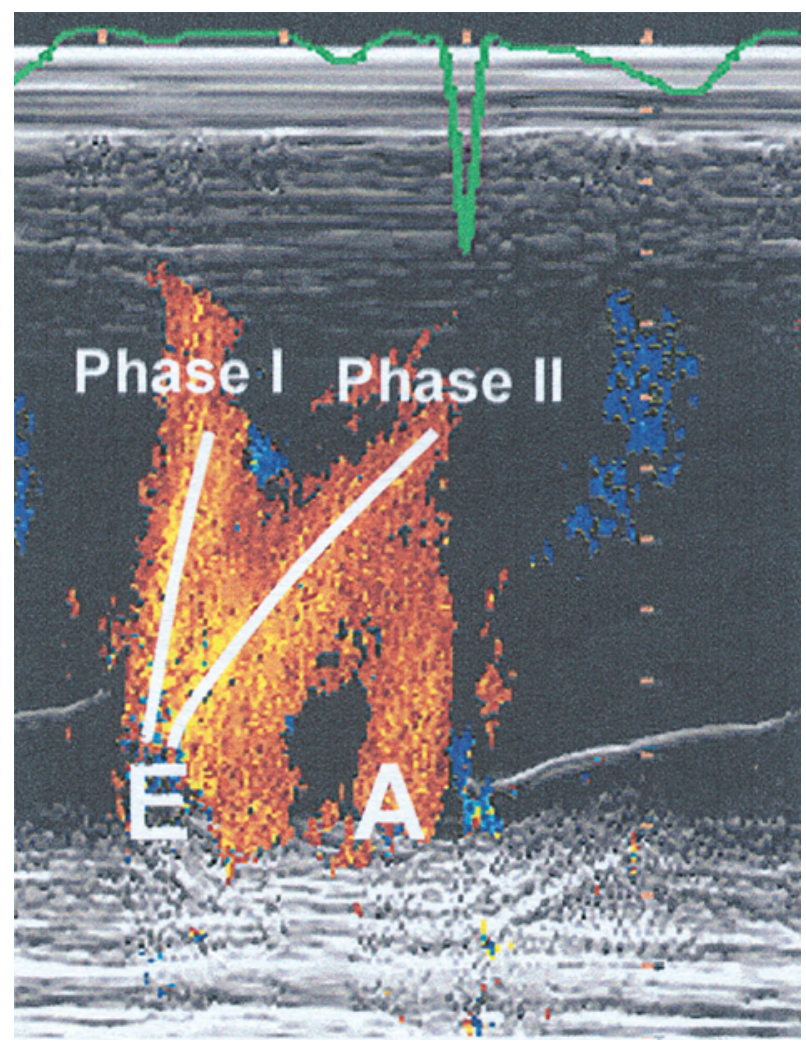

Figure 4. Biphasic early filling wave obtained in a patient using colour M-mode Doppler echocardiography. Phase I is the instantaneous motion of the fluid due to the passing pressure wave, phase II corresponds to the formation and travelling of a vortex ring.

within the red filling flow signals. First the point of maximal velocity around the mitral orifice in early diastole was located. Next, the first aliasing limit was changed to $70 \%$ of the maximal velocity. The nearest point to the apex on the aliasing boundary (typically occurring at the level of the mid ventricle) was then located. The ratio (in $\mathrm{cm} . \mathrm{s}^{-1}$ ) of the distance difference $(S$, in $\mathrm{cm})$ and the time delay $(T$, in $\mathrm{s})$ between the maximal velocity at the mitral valve leaflets and the most apical point of the aliasing boundary corresponding to $70 \%$ of the peak $E$-wave velocity was proposed as a characteristic for $v_{p}$ [Fig. 5(d)]. Note the similarity between the Takatsuji and the Duval-Moulin-Garcia method in using a contour of equal velocities somewhere between zero and maximum velocity. There is, however, an important difference because the DuvalMoulin-Garcia method tracks the front part of the contour to identify a line, whereas Takatsuji et al. uses only one point of the contour (typically at the top), next to the point where the maximum velocity occurs, typically in the centre of the area enclosed by the contour.

Method 5: Greenberg. Greenberg et al. ${ }^{[53]}$ determined $v_{p}$ by eigenvector analysis of the E-wave in the colour M-mode Doppler image. This method calculates the principal components of the E-wave as an elliptical representation. Basically the algorithm is taking into account all velocities in a selected region of interest of the colour M-mode Doppler image and fits an elliptical geometry on it. The angle of rotation of the long axis of the ellipse is used as a parameter for $v_{p}$. This angle is expressed in degrees and negative angles are measured clockwise [Fig. 3(e)].

\section{Reproducibility}

From a theoretical viewpoint, keeping in mind the higher explained relationship between travelling speed of vortices and the position of the maximum velocity along the left ventricular base-apex axis, the approach of Stugaard et al. ${ }^{[49]}$ seems the most straightforward. However, from a practical point of view, other approaches might be preferred for reasons of reproducibility.

Table 1 shows inter- and intra-observer variabilities for the different approaches as reported in literature. Due to difficulties for some images in determining the black-to-colour transition, the method of Brun has a high variability. Also the approach of Stugaard suffers from large variabilities because the method determines the time difference in a semi-automated way, based on only two pixels. None the less, the automated algorithm provides more observer-independent determinations; minor noise in an image can strongly influence the determination of $v_{p}$, yielding large beat-to-beat variabilities. The strict procedure as proposed by Takatsuji et $a l^{[52]}$ has, in case of manual execution, the advantage of filtering out variability. However, analogous to the method of Stugaard, this method finally calculates $v_{p}$ using only two selected pixels, yielding potential sensitivity to noise in an automated procedure, yielding high beat-to-beat variabilities. The values in Table 1 indicate that the Duval-Moulin-Garcia approach has a reproducibility that meets values of standard Doppler indices ${ }^{[54]}$. This justifies the fact that in most of the recent work this approach is used for quantifying $v_{p}$. This method uses more velocity information (all pixels along an isovelocity line) compared to the approaches of Stugaard and Takatsuji and is therefore less noisesensitive. Moreover, linear regression techniques can easily be used for identifying a line with the isovelocity contour, allowing a semiautomated determination of $v_{p}$. The method of Greenberg is a fully automated procedure, taking into account all the velocity information in the selected part of the image. The manual selection of the region of interest remains the only possible source of inter-observer and intra-observer variability. As it integrates all velocity information, the Greenberg method is also almost insensitive to noise in the image. On the other hand, when using the Greenberg method it is hard to unravel whether changes in $v_{p}$ are due to changes in the first or second phase of the ventricular early filling pattern. 
A. Brun

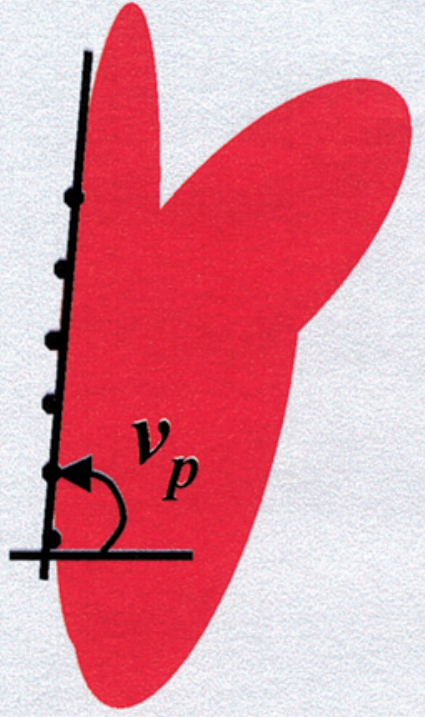

B. Duval-Garcia

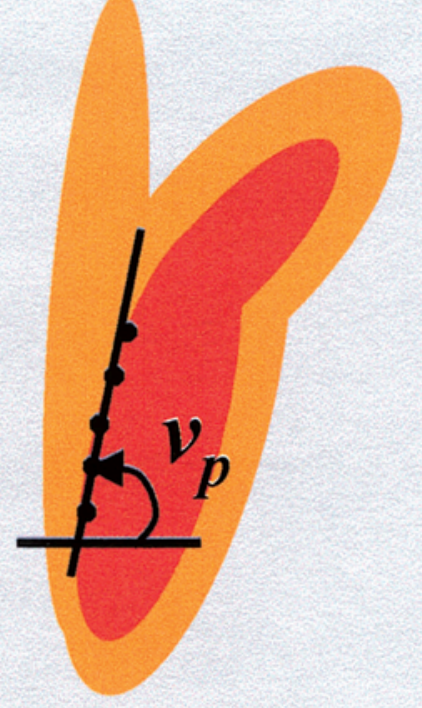

\section{Stugaard}

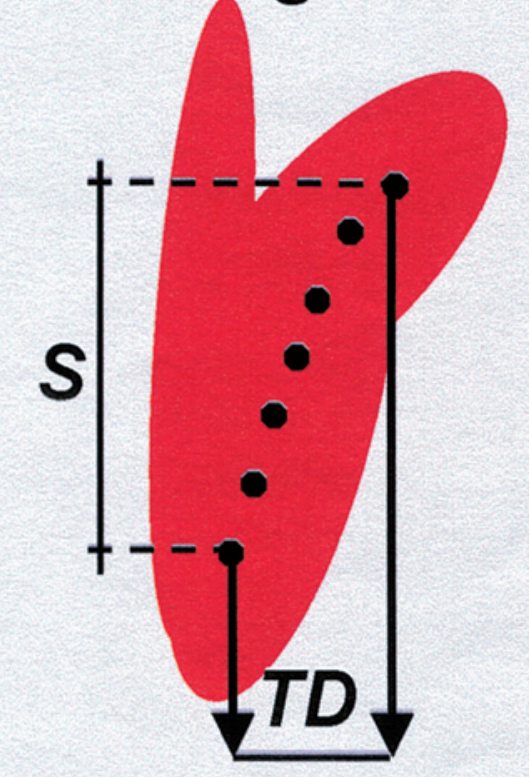

D. Takatsuji E. Greenberg
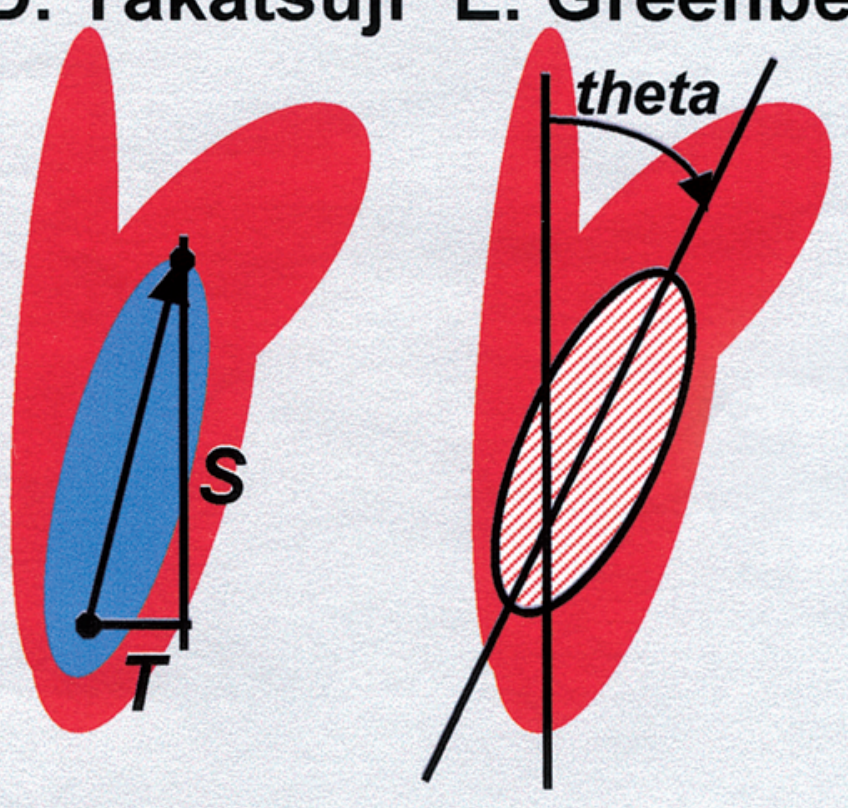

Figure 5. Schematic representation of five different methods for quantifying flow propagation proposed by Brun et al. (a), Duval-Moulin et al. and Garcia et al. (b), Stugaard et al. (c), Takatsuji et al. (d) and Greenberg et al. (e).

\section{Standardization}

Regardless of the approach used, $v_{p}$ is identified with a slope in the spatio-temporal colour M-mode Doppler image. However, the quantification of $v_{p}$ is not standardized. Brun et al. and Takatsuji et al. quantify $v_{p}$ using the tangent of a line, respectively expressed in $\mathrm{m} \cdot \mathrm{s}^{-1}$ and $\mathrm{cm} . \mathrm{s}^{-1}$. In contrast, Stugaard et al. prefer reporting a time delay (TD in ms) or a normalized time delay (TD/S in $\left.\mathrm{ms} . \mathrm{cm}^{-1}\right)$. Greenberg et al. quantifies $v_{p}$ as an angle $\left(\right.$ in $\left.^{\circ}\right)$. As each approach quantifies a slope, transformation of values to a standard quantification is feasible ${ }^{[55]}$. As $v_{p}$ represents the propagation speed of a vortex ring, the reporting of $v_{p}$ as a velocity seems at first sight the most appropriate. However, to improve accuracy when analysing steep slopes, a transformation to an angle 
Table 1. Inter- and intra-observer variability.

\begin{tabular}{|c|c|c|c|c|c|}
\hline \multirow{2}{*}{ Method } & \multicolumn{2}{|c|}{ Inter-observer variability } & \multicolumn{2}{|c|}{ Intra-observer variability } & \multirow{2}{*}{ Reference } \\
\hline & $\%(*)$ & $\mathrm{cm} \cdot \mathrm{s}^{-1}(\dagger)$ & $\%(*)$ & $\mathrm{cm} \cdot \mathrm{s}^{-1}(\dagger)$ & \\
\hline Brun & $18 \pm 18$ & $-1 \cdot 6 \pm 18$ & $20 \pm 9$ & $2 \cdot 7 \pm 10$ & (69) \\
\hline Duval-Moulin-Garcia & $8 \pm 6$ & $1 \cdot 3 \pm 4 \cdot 5$ & $6 \pm 6$ & $2 \cdot 1 \pm 3 \cdot 7$ & (61) \\
\hline Brun & $9 \pm 19$ & NA & $1 \pm 7$ & NA & (57) \\
\hline Duval-Moulin-Garcia & $4 \pm 18$ & NA & $2 \pm 16$ & NA & (48) \\
\hline Duval-Moulin-Garcia & 11 & NA & 9 & NA & (62) \\
\hline Duval-Moulin-Garcia & 12 & NA & 8 & NA & (59) \\
\hline Duval-Moulin-Garcia & $7 \cdot 6 \pm 3 \cdot 8$ & NA & $8 \cdot 3 \pm 6 \cdot 4$ & NA & (87) \\
\hline Duval-Moulin-Garcia & $8 \pm 6$ & NA & $7 \pm 5$ & NA & (68) \\
\hline Brun & $>20$ & NA & $>20$ & NA & (51) \\
\hline Brun & 50 & NA & NA & NA & (56) \\
\hline Stugaard & 20 & NA & NA & NA & (56) \\
\hline Takatsuji & NA & $-1 \cdot 0 \pm 6 \cdot 2$ & NA & $1 \cdot 0 \pm 3 \cdot 4$ & (52) \\
\hline
\end{tabular}

$(*)$ Mean percent error $\pm \mathrm{SD}$; $(\dagger)$ Mean difference $\pm \mathrm{SD}$; NA: not available.

(in ${ }^{\circ}$ ) or a $\log$ transformation might be better. This transformation solves the inaccuracy in case of steep slopes and brings data nearer to homoscedasticity ${ }^{[5,56]}$.

\section{Physiological Determinants of $\mathrm{v}_{\mathrm{p}}$ and $\mathrm{E} / \mathrm{v}_{\mathrm{p}}$}

\section{Age Dependency}

Table 2 shows literature data on normal $v_{p}$ values for different methods. $v_{p}$ is decreasing with age. Agedependency is also observed by Brun et al. ${ }^{[47]}$ and Mego et $a l .{ }^{[57]}$, reporting correlation coefficients of $r=-0.61$ and $r=-0 \cdot 59$ respectively.

\section{Left Ventricular Relaxation}

Studies (Table 3 ) in both animals and humans during coronary angioplasty-induced ischaemia ${ }^{[49,51,58]}$, during intra-coronary dobutamine infusion ${ }^{[47]}$, before and during cardiopulmonary bypass ${ }^{[59]}$, in a mixed patient population ${ }^{[52]}$ and in patients with hypertrophic cardiomyopathy ${ }^{[60]}$ describe a univariate correlation of the constant of isovolumic relaxation (tau) with $v_{p}$ as

Table 2. Normal values of $v_{p}$.

\begin{tabular}{lcccc}
\hline Method & Age & $N$ & $v_{p}$ & Reference \\
\hline Brun & $39 \pm 13$ & 29 & $84 \pm 11$ & $(47)$ \\
Brun & $21-35$ & 21 & $77 \pm 25$ & $(57)$ \\
Brun & 360 & 16 & $70 \pm 23$ & $(57)$ \\
Brun & $51-60$ & 13 & $53 \pm 15$ & $(57)$ \\
Brun & $>65$ & 14 & $43 \pm 11$ & $(57)$ \\
Duval-Moulin-Garcia & $63 \pm 11$ & 30 & $76 \pm 16$ & $(87)$ \\
Duval-Moulin-Garcia & $60 \pm 11$ & 38 & $74 \pm 19$ & $(68)$ \\
Duval-Moulin-Garcia & $37 \cdot 2 \pm 8 \cdot 9$ & 7 & $54 \pm 9$ & $(63)$ \\
Takatsuji & $58 \cdot 2 \pm 13$ & 29 & $74 \pm 17$ & $(52)$ \\
Takatsuji & $56 \pm 10$ & 26 & $73 \pm 19$ & $(60)$ \\
Stugaard & $20-70$ & 54 & $43 \pm 27(*)$ & $(88)$ \\
& & & & \\
\hline
\end{tabular}

Age: mean $\pm \mathrm{SD} ; \mathrm{N}=$ number of studies; $v_{p}=$ flow propagation velocity (mean $\pm \mathrm{SD}$ ) in $\mathrm{cm} \cdot \mathrm{s}^{-1} ;\left(^{*}\right)$ time delay $(T D)$ in $\mathrm{ms}$. obtained from the colour M-mode Doppler images. In both animals and humans, Garcia and co-workers ${ }^{[59]}$ found that left ventricular relaxation is a major determinant of $v_{p}$, best described using a power function tau $=592.21 v_{p}{ }^{-0.6838}(r=-0 \cdot 78, P<0 \cdot 001)$.

\section{Filling Pressure}

A study of Duval-Moulin et al. ${ }^{[51]}$ concludes that during ischaemia, the premature cessation of late filling is associated with increased diastolic pressures. Also, in a univariate analysis, several investigators found a significant correlation between left atrial pressure, left ventricular minimal pressure or left ventricular enddiastolic pressure on one hand and $v_{p}$ on the other hand ${ }^{[47-49,52,58,59,61]}$. In contrast, in multivariate analysis, most studies were not able to demonstrate a significant

Table 3. Relaxation constant and filling pressure as physiological determinants of $\mathrm{v}_{\mathrm{p}}$.

\begin{tabular}{lccc}
\hline Method & r-tau $(*)$ & $\mathrm{r}-\mathrm{p}(\dagger)$ & Reference \\
\hline Animals & & & \\
Duval-Moulin-Garcia & -0.65 & -0.65 & $(59)$ \\
Stugaard & $0 \cdot 81$ & $0 \cdot 78$ & $(49)$ \\
Stugaard & $0 \cdot 89$ & $0 \cdot 91$ & $(58)$ \\
Humans & & & \\
Brun & -0.73 & -0.69 & $(47)$ \\
Takatsuji & -0.82 & -0.61 & $(52)$ \\
Duval-Moulin-Garcia & -0.53 & NA & $(51)$ \\
Takatsuji & -0.76 & NA & $(60)$ \\
Duval-Moulin-Garcia & -0.75 & NA & $(59)$ \\
Duval-Moulin-Garcia & NA & $-0 \cdot 45$ & $(61)$ \\
Duval-Moulin-Garcia & NA & -0.34 & $(48)$ \\
Brun & NA & NS & $(69)$ \\
Duval-Moulin-Garcia & NA & NS & $(63)$ \\
& & & \\
\hline
\end{tabular}

(*) correlation coefficient of the univariate regression between $v_{p}$ (or TD) and the constant of isovolumic relaxation (tau); $(\dagger)$ correlation coefficient of the univariate regression between $v_{p}$ (or $T D$ ) and filling pressure; NA: not available; NS: not significant. 
Normal
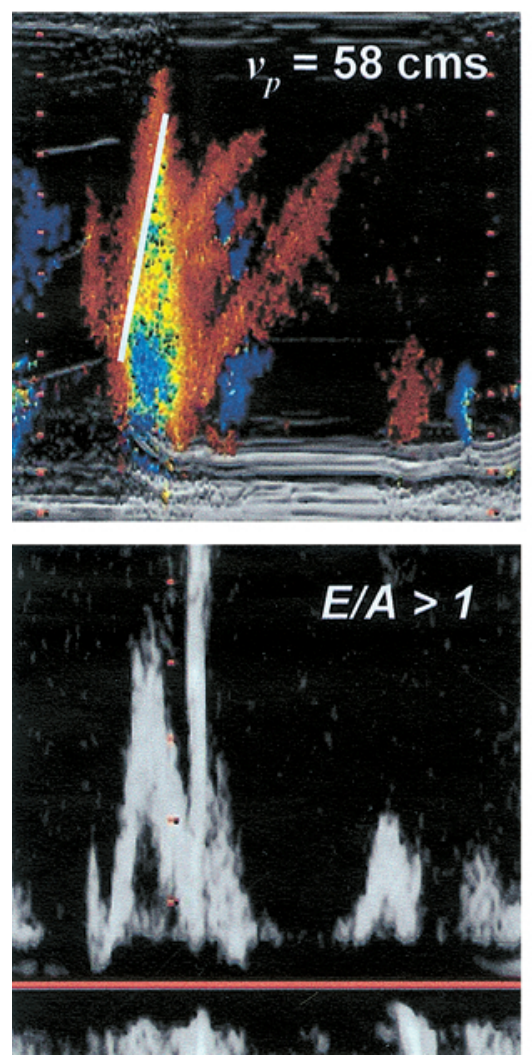

DR
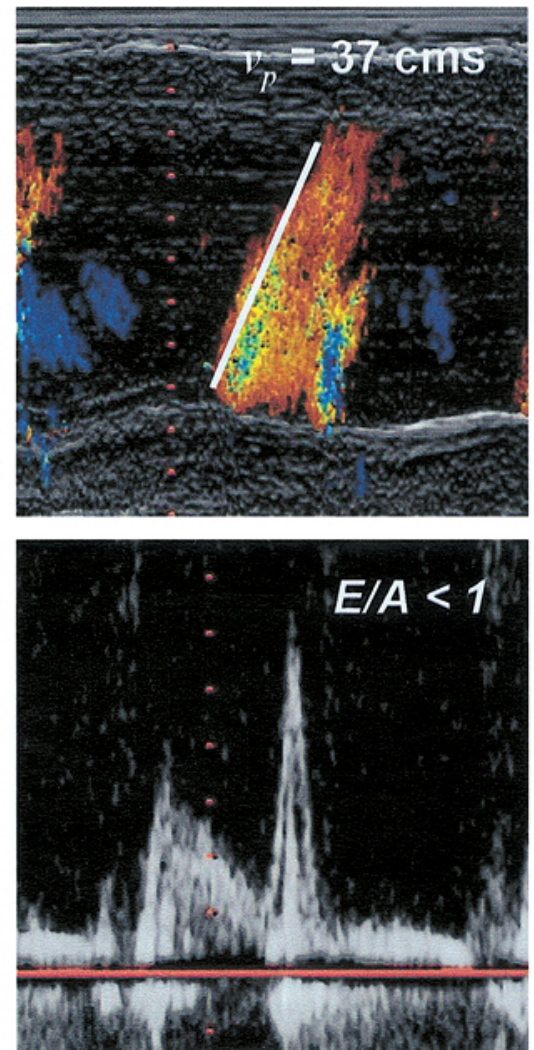

PN
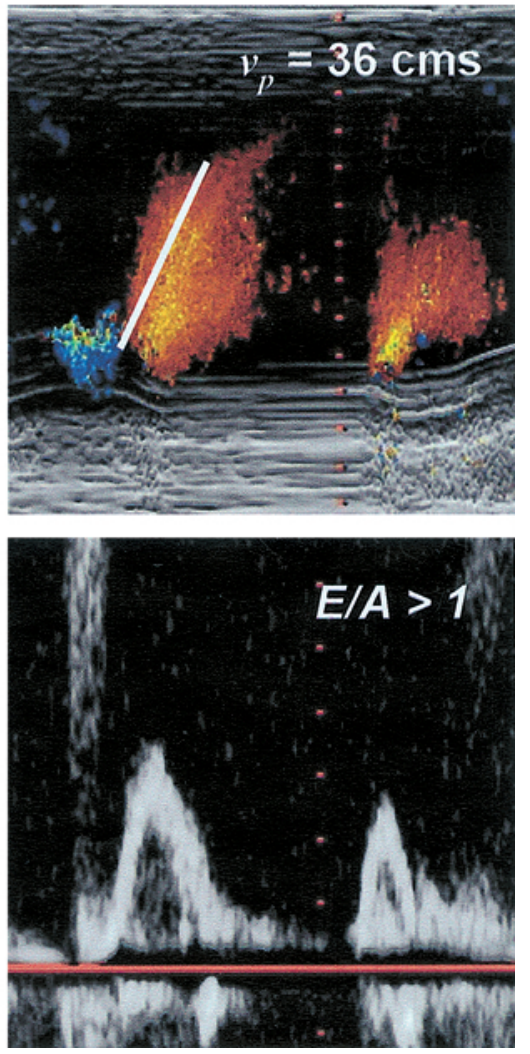

Figure 6. Colour M-mode Doppler and pulsed wave Doppler images of left ventricular filling. Left panel: normal filling pattern $\left(v_{p}=58 \mathrm{~cm} \cdot \mathrm{s}^{-1}, \mathrm{E} / \mathrm{A}>1\right)$. Middle panel: delayed relaxation filling pattern $\left(v_{p}=37 \mathrm{~cm} . \mathrm{s}^{-1}, \mathrm{E} / \mathrm{A}<1\right)$. Right panel: Pseudo-normal filling pattern $\left(v_{p}=36 \mathrm{~cm} \cdot \mathrm{s}^{-1}, \mathrm{E} / \mathrm{A}>1\right)$.

load dependency of $v_{p}$. Only in a study of Brun et al. ${ }^{[47]}$, in a multivariate model, both the relaxation constant and the difference between left ventricular pressure at mitral valve opening and minimal left ventricular pressure remained simultaneously significant variables for determining $v_{p}$. Moreover, several experimental studies $^{[59,62-66]}$ reported no significant change in $v_{p}$ for altering loading conditions including volume loading, caval constriction, Trendelburg's position, inverse Trendelberg's position, after inhalation of amyl nitride and during lower body negative pressure.

\section{Clinical Implications}

In case of diastolic dysfunction, standard pulsed wave Doppler derived indices such as the ratio of peak early filling $(E)$ velocity to peak late filling $(A)$ velocity are sensitive for pseudo-normalization. In contrast, Takatsuji et al. ${ }^{[52]}$ conclude that the spatial and temporal analysis of $v_{p}$ by colour M-mode Doppler echocardiography was free of pseudo-normalization in evaluating left ventricular diastolic dysfunction. This is illustrated in Fig. 6, showing three colour M-mode Doppler images for a normal (normal, left panel), delayed relaxation (DR, middle panel) and pseudo-normal (PN, right panel) filling pattern, next to the corresponding pulsed Doppler tracings of mitral flow. Whereas the $E / A$ ratio can not distinct the normal and the pseudo-normal case, the $v_{p}$ value remains delayed in case of pseudo-normal filling, compared to the normal $v_{p}$ value.

Steine et $a l^{[67]}$ demonstrated slowing of $v_{p}$ in patients with acute myocardial infarction. Infarct size was a determinant of slowing $v_{p}$. In a serial echocardiographic study in patients after a first myocardial infarction, Moller et al. ${ }^{[68]}$ concluded that pseudo-normal or restrictive filling patterns, detected using colour M-mode Doppler, are related to progressive left ventricular dilation and predict cardiac death.

Rajagopalan et al. ${ }^{[50]}$ used colour M-mode to differentiate between constrictive pericardial heart disease and restrictive cardiomyopathy. Their results, obtained using the Duval-Moulin-Garcia method, indicated a progressively slower $v_{p}$ in patients with restrictive cardiomyopathy, compared with patients with constrictive pericarditis. Accordingly the observed time delay, obtained using the approach of Stugaard, was longer in patients with restrictive cardiomyopathy. In contrast, the slope of the black-to-colour transition, as proposed by Brun et al. ${ }^{[47]}$, was not significantly different between the two groups. 
Table 4. Non-invasive estimation of filling pressure using $E / v_{p}$.

\begin{tabular}{llcccc}
\hline Population & \multicolumn{1}{c}{ Method } & $\alpha$ & $\beta$ & $r$ & Reference \\
\hline Atrial fibrilation & Brun & NA & NA & $0 \cdot 65$ & $(69)$ \\
MI & Duval-Moulin-Garcia & $5 \cdot 27$ & $4 \cdot 6$ & $0 \cdot 80$ & $(48)$ \\
HCM & Brun & $5 \cdot 28$ & $4 \cdot 5$ & $0 \cdot 67$ & $(70)$ \\
Miscellaneous & Duval-Moulin-Garcia & NA & NA & $0 \cdot 62$ & $(61)$ \\
Healthy volunteers & Duval-Moulin-Garcia & $0 \cdot 039$ & $1 \cdot 02$ & $0 \cdot 81$ & $(63)$ \\
\hline
\end{tabular}

$\alpha, \beta$ : linear regression model parameters; $r$ : correlation coefficient; MI: myocardial infarction; HCM: hypertrophic cardiomyopathy; NA: not available.

As the main determinants of pulsed wave Doppler peak $E$-velocity are left atrial pressure and left ventricular relaxation, several authors propose the ratio of $E$ and $v_{p}$ as an index of left atrial pressure, left ventricular end-diastolic pressure or pulmonary capillary wedge pressure ${ }^{[48,61,63,69,70]}$. Thus, $v_{p}$ is believed to correct for the load-dependency of $E$. All studies propose a linear relationship of the form:

$$
p=\alpha \cdot \frac{E}{v_{p}}+\beta
$$

where $p$ is filling pressure and $\alpha$ and $\beta$ are fitting constants. Table 4 summarizes these findings. Overall a moderate to excellent correlation is reported $(r=0 \cdot 67-$ 0.87].

Firstenberg et al. ${ }^{[63]}$ reported a relationship between load and $E / v_{p}$ in a population of healthy volunteers. In contrast, Garcia et al. ${ }^{[48]}$ has studied a heterogeneous population of patients. For those two populations, the slope of the relationship between load and $E / v_{p}$ differs significantly. This is an indication that, besides filling pressure, also other covarying left ventricular properties such as relaxation and compliance may interfere in the relationship between load and the $E / v_{p}$ ratio $^{[71]}$.

\section{Pressure Imaging}

\section{Background}

The Navier-Stokes equations are a complex set of coupled differential equations, dictated by the principles of conservation of mass and momentum, and are governing flow velocities. In the situation of the analysis of a central inflow streamline of left ventricular filling, the Navier-Stokes equations can be simplified considerably to the $1 \mathrm{D}$ Euler equation ${ }^{[72]}$ :

$$
\frac{\partial p}{\partial s}=-\rho\left[\frac{\partial v}{\partial t}+v \cdot \frac{\partial v}{\partial s}\right]
$$

where $v(s, t)$ is velocity at time $t$ and distance $s$ along the streamline, $p$ is local pressure, and $\rho$ is blood density. Thus local pressure gradients, $\partial p / \partial s$, can be calculated directly from velocities along a streamline.
The calculation of the partial derivatives of Eq. 3 can be visualized and schematically represented ${ }^{[73]}$. Figure 7 shows this representation for a colour M-mode Doppler image of left ventricular filling. The representation for $\partial v / \partial t$ (time dependent variation of $v$ for all positions along $s$ ) shows first an acceleration (in red), followed by a deceleration (in blue). In the spatial direction $(\partial v / \partial s)$ acceleration is positive in the region of the mitral valve (bottom of the image, blue colour) and deceleration is observed in the upper part of the left ventricle.

\section{Transvalvular Pressure Gradients}

In daily clinical practice, for most stenotic and regurgitant orifices, the simplified Bernoulli equation is commonly used to estimate a pressure difference from measured velocities ${ }^{[17]}$ :

$$
\Delta p=\frac{1}{2} \rho \cdot v^{2}
$$

where $p$ is pressure (in $\mathrm{Pa}$ ), $\rho$ density (in $\mathrm{kg} \cdot \mathrm{m}^{3-1}$ ) of blood and $v$ velocity (in $\mathrm{m} . \mathrm{s}^{-1}$ ). In this equation, the unsteady flow component, the energy losses by turbulence and friction, and the gravitational component are neglected, only retaining the steady component. Filling in the value of the density of blood at $37^{\circ}\left(1060 \mathrm{~kg} . \mathrm{m}^{3-1}\right)$ and converting pressure from $\mathrm{Pa}$ to $\mathrm{mmHg}(0 \cdot 133$ $\mathrm{Pa}=1 \mathrm{mmHg}$ ) results in the equation $\Delta p=4 \cdot v^{2}$. When flow through a normal mitral valve is considered, the assumptions, needed for application of the simplified Bernoulli equation, are not met because of the larger amount of fluid passing through the orifice, yielding a contribution of the unsteady component equalling the steady component. Several authors have addressed the importance of the unsteady component and the according limitations of the simplified Bernoulli equation using in vitro, numerical and experimental studies ${ }^{[18-21,74]}$. Using colour M-mode Doppler echocardiography, by integrating the Euler equation (Eq. 3) the unsteady component of the pressure drop can be included:

$$
\Delta p=\frac{1}{2} \rho \cdot\left(v_{L A}^{2}-v_{L V}^{2}\right)+\rho \int_{L A}^{L V} \frac{\partial v}{\partial t} d s
$$




\section{Pressure Imaging}

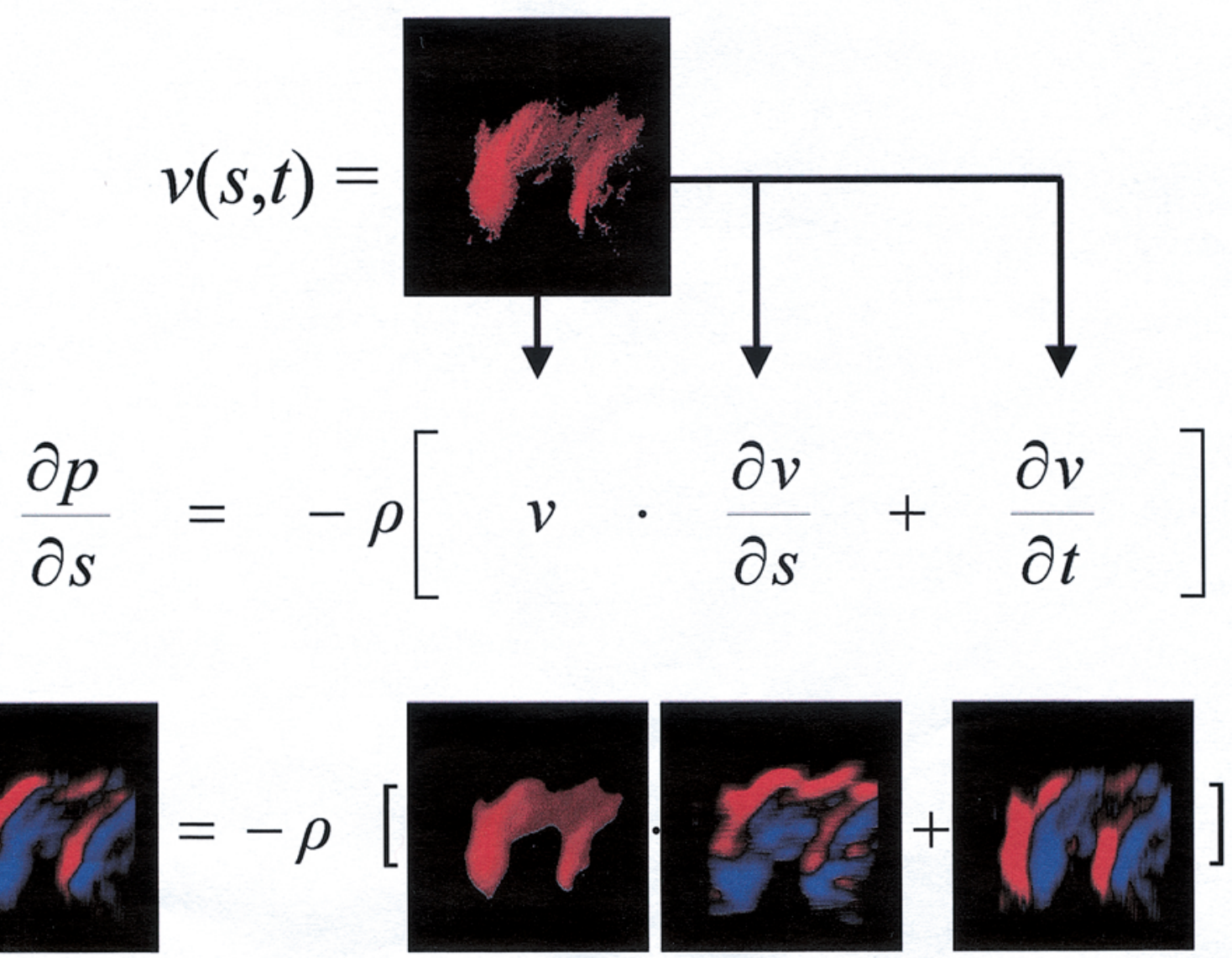

Figure 7. Visual representation of the principles of the pressure imaging technique. Using the spatio-temporal velocity image $v(s, t)$ as an input, spatial $(\partial v / \partial s)$ and temporal $(\partial v / \partial t)$ partial derivatives are calculated and the time dependent pressure gradients $(\partial p / \partial s)$ along the base-apex axis of the left ventricular is reconstructed.

The use of colour M-mode Doppler echocardiography for the estimation of pressure gradients across nonrestrictive valves by integrating the Euler equation (unsteady Bernoulli equation, eq. 5) is validated in our laboratory in a hydraulic model of the left heart, consisting of two elastic chambers separated by a prosthetic biological mitral valve. Simultaneously, high-fidelity LA and left ventricular pressure tracings and Doppler measurements were performed. Water-glycerine mixtures, equalling the viscosity of blood, were used as a test fluid. Pressure drops across the mitral valve were calculated using both the simplified Bernoulli equation and the unsteady Bernoulli equation. Results are illustrated in Fig. 8, confirming that the incorporation of the unsteady flow component using colour M-mode Doppler echocardiography, yields a more accurate estimation of the transvalvular pressure gradient, compared to the simplified Bernoulli equation. Recently, the feasibility of including the unsteady component in the calculation of pressure drops over non-restricted orifices using colour M-mode Doppler echocardiography is demonstrated in $\operatorname{dog} s^{[75]}$ and humans ${ }^{[76,77]}$.

\section{Intra-ventricular Pressure Gradients}

The pressure imaging technique can be extended from transvalvular pressure gradients to intra-ventricular pressure gradients. Intra-ventricular pressure gradients during relaxation and filling of the left ventricle were first described by Ling et al. ${ }^{[78]}$. Courtois et al. ${ }^{[79]}$ observed a significant sub-basal-apical early diastolic pressure gradient along the left ventricular inflow tract with minimum pressure in the apex speculating suction of the blood toward the left ventricular apex. In contrast, using a 1D numerical model, Verdonck et al. ${ }^{[80]}$, and using an axisymmetric three-dimensional model, Vierendeels et al. ${ }^{[46]}$ demonstrated that such pressure gradients are not a rigorous proof of elastic recoil. 


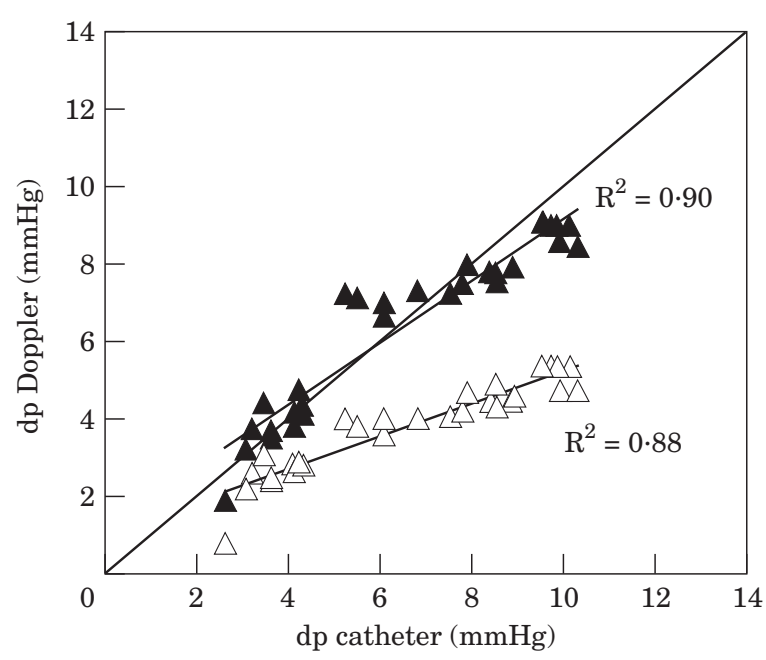

Figure 8. Validation of colour M-mode Doppler derived pressure drops in a hydraulic model of the left heart. Invasive measured pressure drops (x-axis, $\mathrm{mmHg}$ ) vs Doppler derived pressure gradients calculated using the simplified Bernoulli equation (open triangles) and integration of the Euler equation (unsteady Bernoulli equation, closed triangles).

Intra-ventricular pressure gradients yield potential important clinical useful information. In a study of Courtois et al. ${ }^{[79]}$, during acute ischaemia, pressure gradients diminished, making it a possible marker for ischaemia in clinical practice. Recently, Firstenberg et al. demonstrated abrupt improvements in diastolic intra-ventricular gradients following myocardial revascularization $^{[81]}$.

\section{Future Research}

\section{Future Research in Flow Propagation Velocity}

Despite the fact that much basic research has been performed already, the influences of left ventricular properties on the left ventricular filling pattern remains a matter of debate. In the last two decades, numerical and hydraulic models have proved to be very helpful in understanding the detailed physiological underpinnings of the transmitral filling pattern as recorded using pulsed wave Doppler echocardiography. In addition, the development of new or the refinement of existing numerical and hydraulic models that can isolate the influence on the left ventricular filling pattern of individual changes in left ventricular properties can be very useful in unravelling the individual contributions of relaxation, compliance and filling pressure in changes of $v_{p}$ and the $E / v_{p}$ ratio. Preliminary steps in this direction are already undertaken $^{[82]}$.

Whereas in an experimental setting $v_{p}$ measurements are used by several research groups, the daily application in a clinical setting remains difficult, mainly because of a lack in standardization and automation, yielding large operator dependent variabilities. However, the incorporation of standardized and automated algorithms in the analysis tools, provided with the contemporary sonographers, could reduce operator dependent variability and catalyze the introduction of this diagnostic tool in clinical practice.

\section{Future Research in Pressure Imaging}

Intensive research on left ventricular filling using transmitral flow profiles has resulted in several non-invasive approaches for the quantification of left ventricular properties including relaxation and compliance, relying on approximations of the unsteady component of the pressure drop ${ }^{[83,84]}$. The incorporation of the unsteady pressure component can only contribute to an improvement of those methods.

Recently Milde et $a l^{[85]}$ introduced the ventricular diastolic impedance $Z$ as an index of global diastolic function. Global diastolic impedance was defined as left ventricular pressure $P$ over transmitral flow $Q$ during early-rapid filling (E-wave). Simultaneous Doppler E-wave images and micromanometric ventricular pressures were used for calculation of diastolic impedance $Z$. The index $Z$ differentiated between 'impedancematched' (normal diastolic function) and 'impedancemismatched' (abnormal diastolic function) subjects in 19 subjects (10 normal, nine abnormal). Theoretically, longitudinal impedance of left ventricular inflow can be derived non-invasively from colour Doppler M-mode images. Although the feasibility of this technique in a hydraulic model is already demonstrated ${ }^{[82]}$, continuous research remains necessary to unravel the physiological determinants of this index.

The investigation of intra-ventricular pressure gradients is very complicated because it requires complex instrumentation of models, e.g. using multiple high fidelity pressure transducers. This complex setting also limits the application in clinical practice. Recently, however, Greenberg et al. ${ }^{[86]}$ validated the ability of colour M-mode Doppler to quantify these very small pressure gradients and demonstrated their change induced by $\beta$-adrenergic stimulation. The possibility of noninvasively determining intra-ventricular pressure gradients will enhance research possibilities in this field and opens a track for applications in clinical practice.

\section{Conclusion}

Colour M-mode Doppler echocardiography has been shown to be a clinical valuable tool for assessing diastolic function, especially in making a distinction between normal and pseudo-normal filling patterns as well as in the assessment of left atrial pressure. Moreover the colour M-mode Doppler technique has a high potential for new applications in the field of pressure 
imaging. In both fields, the use of automated software is mandatory. With the ever-increasing capacity of computer power, the incorporation of user friendly analysis software on the commercial available sonographers should become reality.

\section{Acknowledgements}

S. De Mey is a recepient of grant IWT-971096 of the Flemish institute for the promotion of scientific-technological research in industry.

\section{References}

[1] Vitarelli A, Gheorghiade M. Diastolic heart failure: standard Doppler approach and beyond. Am J Cardiol 1998; 81: $115 \mathrm{G}-121 \mathrm{G}$

[2] Taylor R, Waggoner A. Doppler assessment of left ventricular diastolic function: a review. J Am Soc Echocardiogr 1992; 5: 603-612.

[3] De Maria A, Wisenbaugh T, Smith M, Harrison M, Berk M. Doppler echocardiographic evaluation of diastolic dysfunction. Circulation 1991; 84 [suppl I]: I-288-I-95.

[4] Nishimura R, Tajik J. Evaluation of diastolic filling of the left ventricle in health and disease: Doppler echocardiography is the clinician's Rosetta stone. $J$ Am Coll Cardiol 1997; 30: 8-18.

[5] Cohen GI, Pietrolungo JF, Thomas JD, Klein AL. A practical guide to assessment of ventricular diastolic function using Doppler echocardiography. J Am Coll Cardiol 1996; 27: $1753-1760$

[6] Flachskampf F, Weyman A, Guerrero J, Thomas J. Calculation of atrioventricular compliance from the mitral flow profile: analytic and in vitro study. J Am Coll Cardiol 1992; 19: 998-1004.

[7] McQueen D, Peskin C, Yellin E. Fluid dynamics of the mitral valve: physiological aspects of a mathematical model. $\mathrm{Am} \mathrm{J}$ Physiol 1982; 242: H1095-H1110.

[8] Meisner J. Left atrial role in left ventricular filling: dog and computer studies ( $\mathrm{PhD}$ thesis). Albert Einstein College of Medicine. New York: Yeshiva University, 1986.

[9] Thomas J, Newel J, Choong C, Weyman A. Physical and physiological determinants of transmitral velocity: numerical analysis. Am J Physiol 1991; 260: H1718-H1730.

[10] Flachskampf F, Rodriguez L, Chen C, Guerrero J, Weyman A, Thomas J. Analysis of Mitral Inertance: a factor critical for early transmitral filling. J Am Soc Echocardiogr 1993; 6: 422-432.

[11] Appleton C. Relation of transmitral flow velocity patterns to left ventricular diastolic function: new insights from a combined hemodynamic and doppler echocardiographic study. J Am Coll Cardiol 1988; 12: 426-440.

[12] Appleton C, Jensen J, Hattle L, Oh J. Doppler evaluation of left and right ventricular diastolic function: a technical guide for obtaining optimal flow velocity recordings. $\mathrm{J} \mathrm{Am} \mathrm{Soc}$ Echocardiogr 1997; 10: 271-291.

[13] Briguori C, Betocchi S, Losi MA et al. Non invasive evaluation of left ventricular diastolic function in hypertrophic cardiomyopathy. Am J Cardiol 1998; 81: 180-187.

[14] Ishida Y, Meisner J, Tsujioka K et al. Left Ventricular filling dynamics: influence of left ventricular relaxation and left atrial pressure. Circulation 1986; 74: 187-196.

[15] Yellin EL, Nikolic SD, Frater RW. Left ventricular diastolic function and mitral valve flow. J Heart Valve Dis 1994; 3: $41-44$

[16] Choong C, Abascal V, Thomas J, Guerrero J, McGlew S, Weyman A. Combined influence of ventricular loading and relaxation on the transmitral flow velocity profile in dogs measured by Doppler echocardiography. Circulation 1988; 78: 672-683.
[17] Hatle L, Angelsen B. Doppler ultrasound in cardiology. Physical principles and clinical applications (2nd edition.): Lea \& Febiger, 1985.

[18] Nakatani S, Firstenberg M, Greenberg N et al. Mitral inertance in humans: critical factor in Doppler estimation of transvalvular pressure gradients. Am J Physiol 2000; 280: H1340-H1345.

[19] Verhaaren H, De Mey S, Coomans I et al. Fixed region of non-distensibility after coarctation repair: in vitro validation of its influence on Doppler peak velocities. $J$ Am Soc Echo 2001; 14: 580-587.

[20] De Mey S, Segers P, Coomans I, Verhaaren H, Verdonck P. Limitations of Doppler echocardiography in the follow-up of aortic coarctation. J Biomech 2001; 34: 951-960.

[21] Baumgartner H, Stefenelli T, Niederberger J, Schima H, Maurer G. 'Overestimation' of catheter gradients by Doppler Ultrasound in patients with aortic stenosis: a predictable manifestation of pressure recovery. J Am Coll Cardiol 1999; 33: $1655-1661$

[22] Appleton C. Hemodynamic determinants of Doppler pulmonary venous flow velocity components: new insights from studies in lightly sedated normal dogs. $J$ Am Coll Cardiol 1997: 30: 1562-1574.

[23] Gulati VK, Katz WE, Follansbee WP, Gorcsan J. Mitral annular descent velocity by tissue Doppler echocardiography as an index of global left ventricular function. Am J Cardiol 1996; 77: 979-984.

[24] Gorcsan Jd, Gulati VK, Mandarino WA, Katz WE. Colorcoded measures of myocardial velocity throughout the cardiac cycle by tissue Doppler imaging to quantify regional left ventricular function. Am Heart J 1996; 131: 1203-1213.

[25] Miyatake K, Yamagishi M, Tanaka N et al. New method for evaluating left ventricular wall motion by color-coded tissue Doppler imaging: in vitro and in vivo studies. $\mathrm{J} \mathrm{Am} \mathrm{Coll}$ Cardiol 1995; 25: 717-724.

[26] Nagueh S, Middleton K, Kopelen H, Zoghbi W, Quinones M. Doppler tissue imaging: a non-invasive technique for evaluation of left ventricular relaxation and estimation of filling pressures. J Am Coll Cardiol 1997; 30: 1527-1533.

[27] Zamorano J, Wallbridge DR, Ge J, Drozd J, Nesser J, Erbel R. Non-invasive assessment of cardiac physiology by tissue Doppler echocardiography. A comparison with invasive haemodynamics. Eur Heart J 1997; 18: 330-339.

[28] Urheim S, Edvardsen T, Torp H, Angelsen B, Smiseth O. Myocardial strain by Doppler echocardiography Validation of a new method to quantify regional myocardial function. Circulation 2000; 102: 1158-1164.

[29] D'hooge J, Heimdal A, Jamal F et al. Regional strain and strain rate measurements by cardiac ultrasound: principles, implementation and limitations. Eur J Echo 2000; 1: 154-170.

[30] Garcia MJ, Thomas JD, Klein AL. New Doppler echocardiographic applications for the study of diastolic function. $J \mathrm{Am}$ Coll Cardiol 1998; 32: 865-875.

[31] Thomas JD, Garcia MJ, Greenberg NL. Application of color Doppler M-mode echocardiography in the assessment of ventricular diastolic function: potential for quantitative analysis. Heart Vessels 1997; 12 (Suppl): 135-137.

[32] Batchelor G. An introduction to fluid dynamics. London: Cambridge University Press, 1967

[33] Lamb H. Hydrodynamics. London: Cambridge University Press, 1932

[34] Lee C, Talbot L. A fluid mechanical study of the closure of heart valves. J Fluid Mech 1979; 91: 41-63.

[35] Bellhouse B. Fluid mechanics of a model mitral valve an left ventricle. Cardiovasc Res 1972; 6: 199-210.

[36] Delemarre B, Visser C, Bot H, AJD. Prediction of apical thrombus formation in acute myocardial infarction based on left ventricular spatial flow pattern. J Am Coll Cardiol 1990; 15: $355-360$.

[37] Van Dantzig JM, Delemarre BJ, Bot H, Koster RW, Visser CA. Doppler left ventricular flow pattern versus conventional predictors of left ventricular thrombus after acute myocardial infarction. J Am Coll Cardiol 1995; 25: 1341-1346. 
[38] Beppu S, Izumi S, Miyatake K. Abnormal blood pathways in left venricular cavity in acute myocardial infarction. Experimental observations with special reference to regional wall motion abnormality and hemostasis. Circulation 1988; 78: $157-164$.

[39] Bot H. Mathematical models of diastolic blood flow patterns in the human left ventricle ( $\mathrm{PhD}$ thesis). Department of Physiology. Amsterdam: Vrij Universiteit, 1989.

[40] Bot H, Verburg J, Delemarre BJ, Strackee J. Determinants of the occurrence of vortex rings in the left ventricle during diastole. J Biomech 1990; 23: 607-615.

[41] Rodevand O, Bjornerheim R, Edvardsen T, Smiseth OA, Ihlen H. Diastolic flow pattern in the normal left ventricle. J Am Soc Echocardiogr 1999; 12: 500-507.

[42] Henry FS, Shortland AP, Iudicello F et al. Flow in a simple model skeletal muscle ventricle: comparison between numerical and physical simulations. J Biomech Eng 1997; 119: 13-19.

[43] Iudicello F, Henry F, Collins M, Salmons S, Sarti A, Lamberti C. Comparison of haemodynamic structures between a skeletal muscle ventricle and the human left ventricle. Internal Medicine 1997; 5: 1-10.

[44] Shortland A. Factors influencing vortex development in a model of a skeletal muscle ventricle. Artificial Organs 1996; 20: 1026-1033.

[45] Steen T, Steen S. Filling of a model left ventricle studied by colour M-mode Doppler. Cardiovasc Res 1994; 28: 1821-1827.

[46] Vierendeels J, Riemslagh K, Dick E, Verdonck P. Computer simulation of intraventricular flow and pressure gradients during diastole. J Biomech Eng 2000; 122: 667-674.

[47] Brun P, Tribouilloy C, Duval AM et al. Left ventricular flow propagation during early filling is related to wall relaxation: a color M-mode Doppler analysis. J Am Coll Cardiol 1992; 20: 420-432.

[48] Garcia MJ, Ares MA, Asher C, Rodriguez L, Vandervoort P, Thomas JD. An index of early left ventricular filling that combined with pulsed Doppler peak E velocity may estimate capillary wedge pressure. J Am Coll Cardiol 1997; 29: 448-454.

[49] Stugaard M, Smiseth OA, Risoe C, Ihlen H. Intraventricular early diastolic filling during acute myocardial ischemia, assessment by multigated color m-mode Doppler echocardiography. Circulation 1993; 88: 2705-2713.

[50] Rajagopalan N, Garcia M, Rodriguez L et al. Comparison of new Doppler echocardiographic methods to differentiate constrictive pericardial heart disease and restrictive cardiomyopathy. Am J Cardiol 2001; 87: 86-94.

[51] Duval-Moulin AM, Dupouy P, Brun P et al. Alteration of left ventricular diastolic function during coronary angioplastyinduced ischemia: a color M-mode Doppler study. J Am Coll Cardiol 1997; 29: 1246-1255.

[52] Takatsuji H, Mikami T, Urasawa K et al. A new approach for evaluation of left ventricular diastolic function: spatial and temporal analysis of left ventricular filling flow propagation by color M-mode Doppler echocardiography. J Am Coll Cardiol 1996; 27: 365-371.

[53] Stugaard M, Greenberg NL, Zhou J, Thomas JD. Automated eigenvector analysis for quantification of color M-mode Doppler filling patterns of the left ventricle in an ischemic canine model. Computers in Cardiology 1997; 24: 61-64.

[54] Galderisi M, Benjamin E, Evans J et al. Intra- and interobserver reproducibility of Doppler-assessed indexes of left ventricular diastolic function in a population-based study (the Framingham heart study). Am J Cardiol 1992; 70: 1341-1346.

[55] De Mey S, Vandervoort P, Pasteuning H, Greenberg N, Verdonck P. Quantification of wave propagation velocity from a colour M-mode Doppler image: implementation and analysis of different methods. Computers in Cardiology 1998; 25: 613-616.

[56] Steen T, Steine K, Smiseth OA, Ihlen H. Repeatability of colour M-mode Doppler measurements of left ventricular filling. Int J Cardiol 1994; 43: 79-85.
[57] Mego DM, DeGeare VS, Nottestad SY et al. Variation of flow propagation velocity with age. J Am Soc Echocardiogr 1998; 11: $20-25$.

[58] Stugaard M, Risoe C, Ihlen H, Smiseth OA. Intracavity filling pattern in the failing left ventricle assessed by color M-mode Doppler echocardiography. J Am Coll Cardiol 1994; 24: 663-670.

[59] Garcia MJ, Smedira NG, Greenberg NL et al. Color M-mode Doppler flow propagation velocity is a preload insensitive index of left ventricular relaxation: animal and human validation. J Am Coll Cardiol 2000; 35: 201-208.

[60] Nishihara K, Mikami T, Takatsuji H et al. Usefulness of early diastolic flow propagation velocity measured by color M-mode doppler technique for the assessment of left ventricular diastolic function in patients with hypertrophic cardiomyopathy. J Am Soc Echocardiogr 2000; 13: 801-808.

[61] Gonzalez-Vilchez F, Ares M, Ayuela J, Alonso L. Combined use of pulsed and color M-mode Doppler echocardiography for the estimation of pulmonary capillary wedge pressure: an empirical approach based on an analytical relation. $J$ Am Coll Cardiol 1999; 34: 515-523.

[62] Garcia MJ, Palac RT, Malenka DJ, Terrell P, Plehn JF. Color M-mode Doppler flow propagation velocity is a relatively preload-independent index of left ventricular filling. $J \mathrm{Am} \mathrm{Soc}$ Echocardiogr 1999; 12: 129-137.

[63] Firstenberg M, Levine B, Garcia M et al. Relationship of echocardiographic indices to pulmonart capillary wedge pressures in healthy volunteers. J Am Coll Cardiol 2000; 36: 1664-1669.

[64] De Mey S, Vandervoort PM, Verdonck PR. Preload (in)dependence of E-wave velocity and wave propagation velocity (abstract). Circulation 1999; 100: 1907.

[65] Moller JE, Poulsen SH, Sondergaard E, Egstrup K. Preload dependence of color M-Mode doppler flow propagation velocity in controls and in patients with left ventricular dysfunction. J Am Soc Echocardiogr 2000; 13: 902-909.

[66] Stugaard M, Smiseth OA, Risoe C, Ihlen H. Intraventricular early diastolic velocity profile during acute myocardial ischemia: a color M-mode Doppler echocardiographic study. J Am Soc Echocardiogr 1995; 8: 270-279.

[67] Steine K, Flogstad T, Stugaard M, Smiseth OA. Early diastolic intraventricular filling pattern in acute myocardial infarction by color M-mode Doppler echocardiography. $J \mathrm{Am}$ Soc Echocardiogr 1998; 11: 119-125.

[68] Moller J, Sondergaard E, Poulsen S, Egstrup K. Pseudonormal and restrictive filling patterns predict left ventricular dilation and cardiac death after a first myocardial infarction: a serial color M-mode Doppler echocardiographic study. J Am Coll Cardiol 2000; 36: 1841-1846.

[69] Nagueh SF, Kopelen HA, Quinones MA. Assessment of left ventricular filling pressures by Doppler in the presence of atrial fibrillation. Circulation 1996; 94: 2138-2145.

[70] Nagueh SF, Lakkis NM, Middleton KJ, Spencer WH, Zoghbi WA, Quinones MA. Doppler estimation of left ventricular filling pressures in patients with hypertrophic cardiomyopathy. Circulation 1999; 99: 254-261.

[71] De Mey S, De Sutter J, Vandervoort PM, Verdonck PR. A new model for the experimental validation of left ventricular filling characteristics (abstract). Circulation 2000; 102: 1775.

[72] Vandervoort PM, Greenberg NL, McCarthy PM, Thomas JD. Estimation of left ventricular filling gradients using digital analysis of color Doppler M-mode velocities. Computers in Cardiology 1993; 20: 293-296.

[73] Greenberg N, Vandervoort P, Thomas J. Noninvasive assessment of diastolic intraventricular pressure gradients using color Doppler M-mode echocardiography. Computers in Cardiology 1995; 22: 1-4.

[74] Pasipoularides A, Murgo J, Miller J, Craig W. Nonobstructive left ventricular ejection pressure gradient in man. Circ Res 1987; 61: 220-227. 
[75] Greenberg NL, Vandervoort PM, Thomas JD. Instantaneous diastolic transmitral pressure differences from color Doppler M mode echocardiography. Am J Physiol 1996; 271: H1267-276.

[76] Firstenberg M, Vandervoort P, Greenberg N et al. Noninvasive estimation of transmitral pressure drop across the normal mitral valve in humans: importance of convective and inertial forces during left ventricular filling. J Am Coll Cardiol 2000; 36: 1942-1949.

[77] Isaaz K. Expanding the frontiers of Doppler echocardiography for the noninvasive assessment of diastolic hemodynamics. J Am Coll Cardiol 2000; 36: 1950-1952.

[78] Ling D, Rankin S, Edwards C, McHale P, Anderson R. Regional diastolic mechanics of the left ventricle in the conscious dog. Am J Physiol 1979; 236: H323-H330.

[79] Courtois M, Kovacs S, Ludbrook P. The transmitral pressureflow velocity relationship: the importance of regional pressure gradients in the left ventricle during diastole. Circulation 1988; 78: 661-671.

[80] Verdonck P, Vierendeels J, Riemslagh K, Dick E. Left ventricular pressure gradients: a computer-model simulation. Med Biol Eng Comput 1999; 37: 1-6.

[81] Firstenberg M et al. Circulation 2001 (in press).

[82] De Mey S, Dumont K, Geeraerts J, Vandervoort P, Verdonck P. Noninvasive assessment of left ventricular longitudinal impedance using color M-mode Doppler echocardiography. Computers in Cardiology 2000; 27: 17-20.
[83] Garcia M, Firstenberg M, Greenberg N et al. Estimation of left ventricular operating stiffness from Doppler early filling deceleration time in humans. Am J Physiol 2001; 280: H554-H561.

[84] Scalia GM, Greenberg NL, McCarthy PM, Thomas JD, Vandervoort PM. Noninvasive assessment of the ventricular relaxation time constant (tau) in humans by Doppler echocardiography. Circulation 1997; 95: 151-155.

[85] Milde J, Sessoms M, Lisauskas J, Bowman A, Courtois M, Kovács S. Ventricular diastolic impedance: a new index of global diastolic function. $J$ Am Coll Cardiol 2000; 35: 185 (abstract)

[86] Greenberg N, Vandervoort P, Firstenberg M, Garcia M, Thomas J. Estimation of diastolic intraventricular pressure gradients by Doppler M-mode echocardiography. Am J Physiol 2001; 280: H2507-H2515.

[87] Moller JE, Sondergaard E, Seward JB, Appleton CP, Egstrup K. Ratio of left ventricular peak E-wave velocity to flow propagation velocity assessed by color M-mode Doppler echocardiography in first myocardial infarction: prognostic and clinical implications. J Am Coll Cardiol 2000; 35: $363-370$.

[88] Stugaard M. Intraventricular filling pattern and diastolic function. Clinical and experimental studies by color M-mode echocardiography (PhD thesis). Medical Department $\mathrm{B}$ and Institute for surgical research. University of Oslo, 1994. 